\title{
Performance Analysis of Orthogonal Frequency Division Multiplexing Based Bidirectional Relay Network in the Presence of Phase Noise
}

\author{
V.N. Senthil Kumaran, S. Rajkumar and S.J. Thiruvengadam \\ Department of Electronics and Communication Engineering, \\ TIFAC CORE in Wireless Technologies, \\ Thiagarajar College of Engineering, Madurai-625 015, India
}

Received 2013-06-20, Revised 2013-08-23; Accepted 2013-09-09

\begin{abstract}
In this study, the performance of OFDM based bidirectional relay network which employs Physical Layer Network Coding (PLNC) is analyzed in the presence of phase noise. The bidirectional relay network is assumed to consist of two sources and a relay, where each node has a single antenna and operates in half duplex mode. The PLNC based OFDM system transmits high data streams over numerous sub channels in frequency domain. Each sub channel is a narrow band flat fading channel, thereby achieving high spectral efficiency over wide band channels. In practice, phase noise is introduced with the information symbols transmitted on all subcarriers in OFDM transceiver. The influence of phase noise on the received signals are analyzed at both the Multiple Access Channel (MAC) and at the Broadcast Channel (BC) environments.
\end{abstract}

Keywords: Bidirectional Relay Network, Physical Layer Network Coding (PLNC), Orthogonal Frequency Division Multiplexing (OFDM), Phase Noise, Multiple Access Channel (MAC), Broadcast Channel (BC)

\section{INTRODUCTION}

In One Way Relay Network (OWRN), the data transmission is unidirectional in which a source node sends the information to the relay and then the relay sends it to the destination (Zhang et al., 2013). Unlike OWRN, in Two Way Relay Network (TWRN), two source terminals exchange their information through a relay node and thereby doubling the data rate. The two of the popular type of modern communications where two source nodes simultaneously send information to each other (Vaze et al., 2011). Many of the TWRN's are analyzed only in the flat fading environments. But most practical wireless communication systems experience the scattered and delayed propagation paths, which is modeled as frequency selective fading channels. One of the methods to analyze the system with frequency selective fading is the OFDM which is capable of converting the way transmission model, first explored by Shannon, is one

frequency selective fading channel into orthogonal flatfading sub channels (Gao et al., 2009; Rabiei et al., 2011; Wang et al., 2010).

Network coding as proposed firstly in (Ahlswede et al., 2000) is a potential and powerful tool in designing communication networks. Network coding has the capability of combining and extracting information's through simple Galois Field additions and multiplications. However, it cannot resolve the interference of simultaneously arriving Electromagnetic (EM) signals at the receiver. Hence, in PLNC the interfering signals are mapped to Galois field additions of two digital bit streams, so that the interference becomes the part of arithmetic operations in network coding (Zhang et al., 2006). In PLNC, the interference at the relay is utilized as selection diversity to improve the throughput performance rather than treating the interference as a degrading factor. A new type of relaying scheme, called partial decode-and-forward was

Corresponding Author: V.N. Senthil Kumaran, Department of Electronics and Communication Engineering, TIFAC CORE in Wireless Technologies, Thiagarajar College of Engineering, Madurai-625 015, India 
designed for TWRC with fading, along with space-time codes that achieves full spatial diversity. The optimal mapping functions at the relay nodes that could minimize the transmission Bit Error Rate (BER) were proposed in (Ding et al., 2009).

In OFDM systems, the degradation with orthogonality due to the phase noise is the major issue. The effect of Phase Noise (PHN) on the OFDM signal is modeled in (Tomba, 1998), where PHN causes a rotation of each time-domain OFDM sample by a random phase drift. The sensitivity of the phase noise of the oscillator used for frequency down-conversion is discussed in (Armada, 2001). The effect of phase noise in the presence of multipath fading environment is discussed in (Wu and Bar-Ness, 2004). The influence of phase noise on OFDM is analyzed in (Petrovic et al., 2007; Rabiei et al., 2008).

In this study, the effect of phase noise on the OFDM based bidirectional relay network is analyzed and the tight upper/lower bounds for average symbol error probability is also obtained. The degradation in the bit error rate performance caused by the presence of carrier phase noise is analytically evaluated. The max-log approximation is adopted and the ML detection rule is approximated to make the performance analysis tractable (MinChul and Kim, 2010).

The rest of the study is organized as follows. The system model in the presence of phase noise is presented. In this study the performance of the system based on signal to interference noise ratio and bit error rate are analyzed. Outage performance is also analyzed at both low SNR and high SNR regimes. The simulation results are discussed and finally the concluding remarks are addressed and summarized.

\section{MATERIALS AND METHODS}

\subsection{System Model for OFDM Based Bidirectional Relay Network}

Consider a bidirectional relay network consisting of two nodes $\mathrm{S}_{1}, \mathrm{~S}_{2}$ and a relay node $\mathrm{R}$ where each node has a single antenna and operates in a half duplex mode as shown in Fig. 1. The channels between $S_{1}$ to $R$ and $S_{2}$ to $\mathrm{R}$ are assumed to be quasi-static frequency selective fading such that the channel statistics does not change within a transmission duration of one frame but can vary from one frame to another. The baseband equivalent channel impulse response between $\mathrm{S}_{1}$ and $\mathrm{R}$ is denoted by $h=\left[h_{0}, h_{1}, \ldots, h_{L_{1}-1}\right], \quad h_{i}{ }^{\prime} s \quad$ are independent and identically distributed (iid) complex Gaussian with zero mean and variance $\sigma_{h}^{2}$. The channel between $S_{2}$ and $R$ is denoted by $\mathbf{g}=\left[\mathrm{g}_{0}, \mathrm{~g}_{1}, \ldots, \mathrm{g}_{\mathrm{L}_{2}-1}\right] \mathrm{g}_{\mathrm{i}}$ 's are independent and identically distributed (iid) complex Gaussian with zero mean and variance $\sigma_{\mathrm{g}}^{2} . \mathrm{L}_{1}$ and $\mathrm{L}_{2}$ represent the number of taps of the corresponding channel. The frequency response of the channel impulse response vectors $h$ and $g$ are given by $\mathrm{w}_{\mathrm{h}}[\mathrm{k}]=\sum_{\mathrm{l}=0}^{\mathrm{L}_{1}-1} \mathrm{~h}[1] \mathrm{e}^{\frac{-\mathrm{j} 2 \pi \mathrm{kl}}{\mathrm{N}}}, \mathrm{k}=0,1,2, \ldots \mathrm{N}-1$ and $\mathrm{w}_{\mathrm{g}}[\mathrm{k}]=\sum_{\mathrm{l}=0}^{\mathrm{L}_{2}-1} \mathrm{~g}[1] \mathrm{e}^{\frac{-\mathrm{j} 2 \pi \mathrm{kl}}{\mathrm{N}}}, \mathrm{k}=0,1,2, \ldots \mathrm{N}-1$.

During Phase I, both the nodes $\mathrm{S}_{1}$ and $\mathrm{S}_{2}$ transmit OFDM signals with $\mathrm{N}$ information symbols $\tilde{\mathbf{x}}_{\mathbf{j}}=\left[\tilde{\mathrm{x}}_{\mathrm{j}}[0], \tilde{\mathrm{x}}_{\mathrm{j}}[1], \ldots, \tilde{\mathrm{x}}_{\mathrm{j}}[\mathrm{N}-1]\right.$, where $\mathrm{j}=1,2$ and a Cyclic Prefix (CP) of length $\mathrm{L}_{\mathrm{CP}}$, to the relay node $\mathrm{R}$ via Multiple Access Channel (MAC). The CP length $\mathrm{L}_{\mathrm{CP}}$ satisfies the condition that $\mathrm{L}_{\mathrm{CP}} \geq \max \left\{\mathrm{L}_{1}-1, \mathrm{~L}_{2}-1\right\}$ to avoid Inter-Symbol Interference (ISI). In an ideal case, the transmitted symbols on all subcarriers $\mathrm{k}=0,1,2, . . \mathrm{N}-1$ can be ideally recovered from the received signal samples. But, in practice, phase noise may present at both the source nodes $S_{1}, S_{2}$ and at the relay node. The phase noise causes significant degradation in the performance of OFDM based wireless systems. At the receiver side, due to phase noise, the effective Signalto-Noise Ratio (SNR) is reduced. It increases the Bit Error Rate (BER) and decreases the data rate. The spectral components of phase noise that contribute to the magnitude of the error cause the intersymbol interference. It varies from subcarrier spacing, $\Delta \mathrm{f}$ to the total phase noise bandwidth. When the phase noise bandwidth is greater than the subcarrier spacing, then the intercarrier interference dominates over the Common Phase Error (CPE). The phase noise at $\mathrm{k}^{\text {th }}$ subcarrier is given by Equation (1):

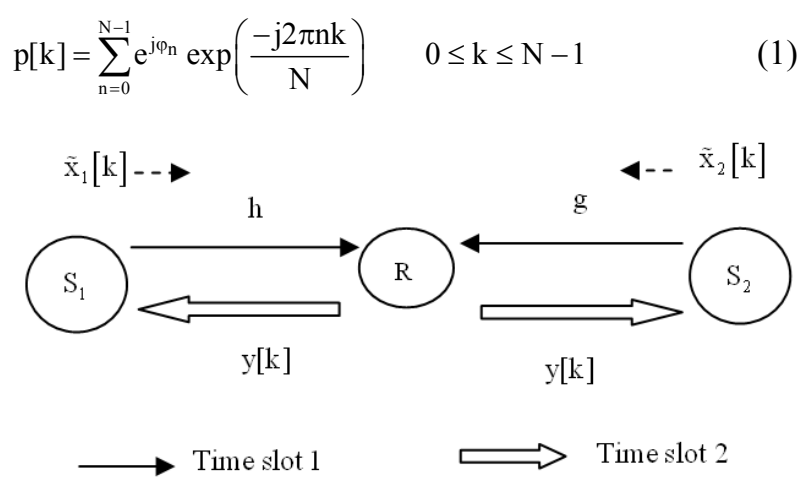

Fig. 1. System model 
The time domain phase noise component, $\phi_{\mathrm{n}}$ is modeled as $\phi_{\mathrm{n}}=\phi_{\mathrm{n}-1}+\varepsilon$, with the initial condition $\phi_{0}=0$ and Wiener process $\varepsilon \sim \mathrm{N}\left(0, \sigma_{\varepsilon}^{2}\right)$. The phase noise variance, $\sigma_{\varepsilon}^{2}=2 \pi \beta \mathrm{T}$, where $\beta$ is the phase noise bandwidth and $\mathrm{T}$ is the OFDM symbol duration. Assuming that the phase noise bandwidth is smaller, relative to the subcarrier spacing, it is assumed that perfect timing and frequency offset correction is performed. The received signal at the relay after taking DFT, is represented as Equation (2):

$$
\begin{array}{rl}
\tilde{r}[k]= & {\left[\sqrt{E_{1}} \tilde{x}_{1}[k] w_{h}[k]+\sqrt{E_{2}} w_{g}[k] \tilde{x}_{2}[k] w_{g}[k]\right] p[0]} \\
& +\left[\sum_{q=0, q \neq k}^{N-1} \tilde{x}_{1}[q] w_{h}[q]+\sum_{q=0, q \neq k}^{N-1} \tilde{x}_{2}[q] w_{g}[q]\right] p_{r}(q-k) \\
& +n_{r}[k] \\
k=0 & 0,1, \ldots N-1 \text { and } \mathrm{q}=0,1, \ldots N-1
\end{array}
$$

\section{Where:}

$\mathrm{E}_{1}$ and $\mathrm{E}_{2}=$ The transmission powers at nodes $\mathrm{S}_{1}$ and $\mathrm{S}_{2}$ respectively

$\mathrm{n}_{\mathrm{r}}[\mathrm{k}]=$ Zero mean circularly symmetric complex Gaussian noise with variance

$\sigma_{\mathrm{r}}^{2}, \mathrm{p}[0]=$ The Common Phase Error (CPE) and $\mathrm{p}(\mathrm{q}-$ $\mathrm{k}$ ) is the Inter-Carrier Interference (ICI) due to the phase noise.

The phase noise element $\mathrm{p}[\mathrm{k}]$ at the relay affects the received signals as an angular multiplicative distortion in the time domain. The relay detects the received symbols at subcarriers $\mathrm{k}=0,1,2, \ldots, \mathrm{N}-1$. The transmitted symbol sequences $\tilde{\mathbf{x}}_{1}$ and $\tilde{\mathbf{x}}_{2}$ are jointly estimated using Maximum Likelihood (ML) principle at $\mathrm{R}$. The estimate of the $\mathrm{k}^{\text {th }}$ symbols of both the sequence is given by Equation (3):

$$
\begin{aligned}
& \left\{\hat{\mathrm{x}}_{1}[\mathrm{k}], \hat{\mathrm{x}}_{2}[\mathrm{k}]\right\}= \\
& \underset{\left(\tilde{\mathrm{x}}_{1}(\mathrm{k}), \tilde{\mathrm{x}}_{2}(\mathrm{k})\right) \in \mathrm{A}^{2}}{\arg \min }\left|\tilde{\mathrm{r}}[\mathrm{k}]-\mathrm{w}_{\mathrm{h}}[\mathrm{k}] \tilde{\mathrm{x}}_{1}[\mathrm{k}]-\mathrm{w}_{\mathrm{g}}[\mathrm{k}] \tilde{\mathrm{x}}_{2}[\mathrm{k}]\right|^{2}, \\
& \quad \mathrm{k}=0,1, \ldots \mathrm{N}-1
\end{aligned}
$$

where, $\mathrm{A}^{2}$ is the second order extension of the alphabet $A \in\{1,-1\}$. Using the joint estimation of the transmitted sequences, a new sequence $\hat{\mathrm{x}}_{3}[\mathrm{k}]$ is formed as $\hat{\mathrm{x}}_{3}[\mathrm{k}]=\hat{\mathrm{x}}_{1}[\mathrm{k}] \hat{\mathrm{x}}_{2}[\mathrm{k}], \mathrm{k}=0,1 \ldots \mathrm{N}-1$. At the relay, OFDM signal corresponding to the signal vector $\hat{\mathrm{x}}_{3}[\mathrm{k}]$ is generated by taking IDFT and appending CP of length $\mathrm{L}_{\mathrm{CP}}$. In Phase II, the relay, $\mathrm{R}$ broadcasts the OFDM signal to the destination nodes $\mathrm{S}_{1}$ and $\mathrm{S}_{2}$ over the broadcast channel. Assuming perfect timing and frequency offset, the received signal at destinations $\mathrm{y}_{1}[\mathrm{k}]$ and $\mathrm{y}_{2}[\mathrm{k}]$ at the source nodes $\mathrm{S}_{1}$ and $\mathrm{S}_{2}$ perturbed by phase noise is given by Equation $(4,5)$ :

$$
\begin{aligned}
\mathrm{y}_{1}[\mathrm{k}] & =\left[\sqrt{\mathrm{E}_{\mathrm{r}}} \hat{\mathrm{x}}_{3}[\mathrm{k}] \mathrm{w}_{\mathrm{h}}[\mathrm{k}]\right] \mathrm{p}[0] \\
+ & {\left[\sum_{\mathrm{q}=0, \mathrm{q} \neq \mathrm{k}}^{\mathrm{N}-1} \sqrt{\mathrm{E}_{\mathrm{r}}} \hat{\mathrm{x}}_{3}[\mathrm{k}] \mathrm{w}_{\mathrm{h}}[\mathrm{k}]\right] \mathrm{p}_{1}[\mathrm{q}-\mathrm{k}] } \\
+\tilde{\mathrm{n}}_{1}[\mathrm{k}] & \\
\mathrm{y}_{2}[\mathrm{k}] & =\left[\sqrt{\mathrm{E}_{\mathrm{r}}} \hat{\mathrm{x}}_{3}[\mathrm{k}] \mathrm{w}_{\mathrm{g}}[\mathrm{k}]\right] \mathrm{p}[0] \\
+ & {\left[\sum_{\mathrm{q}=0, \mathrm{q} \neq \mathrm{k}}^{\mathrm{N}-1} \sqrt{\mathrm{E}_{\mathrm{r}}} \hat{\mathrm{x}}_{3}[\mathrm{k}] \mathrm{w}_{\mathrm{g}}[\mathrm{k}]\right] \mathrm{p}_{2}[\mathrm{q}-\mathrm{k}] } \\
+ & \tilde{\mathrm{n}}_{2}[\mathrm{k}]
\end{aligned}
$$

where, $\mathrm{E}_{\mathrm{r}}$, is the transmission power at $\mathrm{R}$ and $\mathrm{p}_{1}(\mathrm{q}-\mathrm{k})$, $\mathrm{p}_{2}(\mathrm{q}-\mathrm{k})$ are the Inter-carrier interference due to the phase noise. $\tilde{\mathrm{n}}_{1}[\mathrm{k}]$ and $\tilde{\mathrm{n}}_{2}[\mathrm{k}]$ are circularly symmetric complex Gaussian noises with zero mean and variances $\sigma_{\mathrm{n}, 1}^{2}$ and $\sigma_{\mathrm{n}, 2}^{2}$ respectively. The ML estimate of $\tilde{\mathrm{x}}_{2}[\mathrm{k}]$ and $\tilde{\mathrm{x}}_{1}[\mathrm{k}]$ at $S_{1}$ and $S_{2}$ respectively, are given by Equation $(6,7)$ :

$$
\begin{aligned}
& \hat{\mathrm{x}}_{2}[\mathrm{k}]=\underset{\mathrm{x}_{2}[\mathrm{k}] \in \mathrm{A}}{\arg \min }\left|\mathrm{y}_{1}[\mathrm{k}]-\sqrt{\mathrm{E}_{\mathrm{r}}} \mathrm{w}_{\mathrm{h}}[\mathrm{k}] \tilde{\mathrm{x}}_{1}[\mathrm{k}]\right|^{2} \\
& \hat{\mathrm{x}}_{1}[\mathrm{k}]=\underset{\mathrm{x}_{1}[\mathrm{k}] \in \mathrm{A}}{\arg \min }\left|\mathrm{y}_{2}[\mathrm{k}]-\sqrt{\mathrm{E}_{\mathrm{r}}} \mathrm{w}_{\mathrm{g}}[\mathrm{k}] \tilde{\mathrm{x}}_{2}[\mathrm{k}]\right|^{2}
\end{aligned}
$$

Then, $\mathrm{S}_{1}$ estimates $\hat{\mathrm{x}}_{2}[\mathrm{k}]$ by multiplying the symbol $\hat{y}_{1}[\mathrm{k}]$ with its own symbol $\tilde{\mathrm{x}}_{1}[\mathrm{k}]$ and $\mathrm{S}_{2}$ estimates $\hat{\mathrm{x}}_{1}[\mathrm{k}]$ by multiplying the symbol $\hat{y}_{2}[\mathrm{k}]$ with its own symbol $\tilde{\mathrm{x}}_{2}[\mathrm{k}]$ as follows Equation $(8,9)$ :

$$
\begin{aligned}
& \hat{\mathrm{x}}_{2}[\mathrm{k}]=\hat{\mathrm{y}}_{1}[\mathrm{k}] \tilde{\mathrm{x}}_{1}[\mathrm{k}] \\
& \hat{\mathrm{x}}_{1}[\mathrm{k}]=\hat{\mathrm{y}}_{2}[\mathrm{k}] \tilde{\mathrm{x}}_{2}[\mathrm{k}]
\end{aligned}
$$

\subsection{SINR Analysis}

The Signal-to-Interference Noise Ratio (SINR) expressions for MAC and $\mathrm{BC}$ transmission modes are derived. It is assumed that the CPE term has been compensated. From Equation (2), the instantaneous SINRs for the signals from $\mathrm{S}_{1}$ and $\mathrm{S}_{2}$ at $\mathrm{k}^{\text {th }}$ subcarrier, conditioned on the individual channel knowledge, are derived as Equation (10 and 11): 


$$
\begin{aligned}
& \gamma_{1}[\mathrm{k}]=\frac{\varepsilon_{\mathrm{x}_{1}}\left|\mathrm{w}_{\mathrm{h}}[\mathrm{k}]\right|^{2}}{\varepsilon_{\mathrm{x}_{1}}\left|\sum_{\mathrm{q}=0, \mathrm{q} \neq \mathrm{k}}^{\mathrm{N}-1} \mathrm{P}(\mathrm{k}-\mathrm{q}) \mathrm{w}_{\mathrm{h}}[\mathrm{k}]\right|^{2}+\sigma_{\mathrm{r}}^{2}} \\
& \gamma_{2}[\mathrm{k}]=\frac{\varepsilon_{\mathrm{x}_{2}}\left|\mathrm{w}_{\mathrm{g}}[\mathrm{k}]\right|^{2}}{\varepsilon_{\mathrm{x}_{2}}\left|\sum_{\mathrm{q}=0, \mathrm{q} \neq \mathrm{k}}^{\mathrm{N}-1} \mathrm{P}(\mathrm{k}-\mathrm{q}) \mathrm{w}_{\mathrm{g}}[\mathrm{k}]\right|^{2}+\sigma_{\mathrm{r}}^{2}}
\end{aligned}
$$

where, $\varepsilon_{\mathrm{x}_{1}}=\mathrm{E}\left(\left|\tilde{\mathrm{x}}_{1}[\mathrm{k}]\right|^{2}\right)$ and $\varepsilon_{\mathrm{x}_{2}}=\mathrm{E}\left(\left|\tilde{\mathrm{x}}_{2}[\mathrm{k}]\right|^{2}\right)$.

Using Cauchy's Schwartz inequality, the denominator term is written as Equation (12):

$$
\left|\sum_{\mathrm{q}=0, \mathrm{q} \neq \mathrm{k}}^{\mathrm{N}-1} \mathrm{P}(\mathrm{k}-\mathrm{q}) \mathrm{w}_{\mathrm{h}}(\mathrm{q})\right|^{2} \leq \sum_{\mathrm{n}=1}^{\mathrm{N}-1}|\mathrm{P}(\mathrm{n})|^{2} \sum_{\mathrm{m}=1}^{\mathrm{N}-1}\left|\mathrm{w}_{\mathrm{h}}(\mathrm{m})\right|^{2}
$$

In (12), the phase noise component can be written as Equation (13):

$$
\sum_{n=1}^{N-1}|P(n)|^{2}=\left(1-\sigma_{p}^{2}\right) \text { with } \sigma_{p}^{2}=E\left(|P(0)|^{2}\right)
$$

Substituting (13) in (12), it is rewritten as Equation (14):

$$
\left|\sum_{\mathrm{q}=0, \mathrm{q} \neq \mathrm{k}}^{\mathrm{N}-1} \mathrm{P}(\mathrm{k}-\mathrm{q}) \mathrm{w}_{\mathrm{h}}(\mathrm{q})\right|^{2} \leq\left(1-\sigma_{\mathrm{p}}^{2}\right) \sum_{\mathrm{m}=1}^{\mathrm{N}-1}\left|\mathrm{w}_{\mathrm{h}}(\mathrm{m})\right|^{2}
$$

Substituting (14) in (10) Equation (15):

$$
\gamma_{1}[\mathrm{k}] \geq \frac{\left|\mathrm{w}_{\mathrm{h}}(\mathrm{k})\right|^{2}}{\left(1-\sigma_{\mathrm{p}}^{2}\right)\left|\sum_{\mathrm{q}=0, \mathrm{q} \neq \mathrm{k}}^{\mathrm{N}-1} \mathrm{w}_{\mathrm{h}}(\mathrm{q})\right|^{2}+\frac{\sigma_{\mathrm{r}}^{2}}{\varepsilon_{\mathrm{x}_{1}}}},
$$

For small phase noise, the variance, $\sigma_{\mathrm{p}}^{2}$ of the CPE term $[\mathrm{p}(0)]$ i.e., at $\mathrm{k}=0$ can be expressed as follows, From (Wu and Bar-Ness, 2004), the energy of phase noise component is expressed as:

$$
\mathrm{E}\left[|\mathrm{P}(\mathrm{k})|^{2}\right]=\frac{1}{\mathrm{~N}^{2}}\left\{2 \mathfrak{R}\left(\frac{\mathrm{d}_{\mathrm{k}}^{\mathrm{N}+1}-(\mathrm{N}+1) \mathrm{d}_{\mathrm{k}}+\mathrm{N}}{\left(\mathrm{d}_{\mathrm{k}}-1\right)^{2}}\right)-\mathrm{N}\right\}
$$

Where:

$$
d_{k}=e^{j\left(\frac{2 \pi k}{N}\right)-\left(\frac{\pi \beta}{R}\right)}
$$

Since, the phase noise variance, $\sigma_{\mathrm{phn}}^{2}=2 \pi \beta \mathrm{T}_{\mathrm{s}}=2 \pi \beta / \mathrm{R}_{\mathrm{d}}$ with data rate, $\mathrm{R}_{\mathrm{d}}=1 / \mathrm{T}_{\mathrm{s}}$. Formulating for $\mathrm{x}=\frac{-\sigma_{\mathrm{phn}}^{2}}{2}$, we get $\mathrm{x}=-\frac{\pi \beta}{\mathrm{R}_{\mathrm{d}}} \quad$ and for $\mathrm{x}=-\frac{(\mathrm{N}+1) \sigma_{\mathrm{phn}}^{2}}{2}$, we get $\mathrm{x}=-\frac{(\mathrm{N}+1) \pi \beta}{\mathrm{R}_{\mathrm{d}}}$. After several manipulations, the variance of the phase noise term is:

$$
\begin{aligned}
& \mathrm{E}\left[|\mathrm{P}(\mathrm{k})|^{2}\right]= \\
& \frac{1}{\mathrm{~N}^{2}}\left[\frac{(\mathrm{N}+1)\left\{\frac{-(\mathrm{N}+1)^{2} \sigma_{\mathrm{phn}}^{2}}{6}+\frac{\sigma_{\mathrm{phn}}^{2}}{6}+\mathrm{N}\right\}}{\left(1-\frac{\sigma_{\mathrm{phn}}^{2}}{4}+\frac{\sigma_{\mathrm{phn}}^{4}}{24}\right)^{2}}-\mathrm{N}\right](\because \mathrm{k}=0)
\end{aligned}
$$

Neglecting the higher order moments the phase noise variance is simplified as:

$$
\begin{aligned}
E\left[|\mathrm{P}(\mathrm{k})|^{2}\right] & = \\
\frac{1}{\mathrm{~N}^{2}}[(\mathrm{~N}+1) & \left.\left\{\frac{-(\mathrm{N}+1)^{2} \sigma_{\mathrm{phn}}^{2}}{6}+\frac{\sigma_{\mathrm{phn}}^{2}}{6}+\mathrm{N}\right\}-\mathrm{N}\right] \\
= & \frac{1}{6 \mathrm{~N}}\left(6 \mathrm{~N}-\sigma_{\mathrm{phn}}^{2}(\mathrm{~N}+1)(\mathrm{N}+2)\right) \\
= & {\left[1-\frac{\sigma_{\mathrm{phn}}^{2}(\mathrm{~N}+1)(\mathrm{N}+2)}{6 \mathrm{~N}}\right] }
\end{aligned}
$$

Hence, $\sigma_{p}^{2}=1-\frac{\pi \beta N}{3 R_{d}}$, where $R_{d}$ is the data rate.

Finally $\gamma_{1}[\mathrm{k}]$ and $\gamma_{2}[\mathrm{k}]$ can be written as Equation (16):

$\gamma_{1}[\mathrm{k}] \geq \frac{\left|\mathrm{w}_{\mathrm{h}}(\mathrm{k})\right|^{2}}{\frac{\pi \beta N T_{\mathrm{s}}}{3}\left|\sum_{\mathrm{q}=0, \mathrm{q} \neq \mathrm{k}}^{\mathrm{N}-1} \mathrm{w}_{\mathrm{h}}(\mathrm{q})\right|^{2}+\frac{\sigma_{\mathrm{r}}^{2}}{\varepsilon_{\mathrm{x}_{1}}}}$,

and similarly the SINR at the destination $\mathrm{S}_{2}$ is given as Equation (17):

$$
\gamma_{2}[\mathrm{k}] \geq \frac{\left|\mathrm{w}_{\mathrm{g}}(\mathrm{k})\right|^{2}}{\frac{\pi \beta N T_{\mathrm{s}}}{3}\left|\sum_{\mathrm{q}=0, \mathrm{q} \neq \mathrm{k}}^{\mathrm{N}-1} \mathrm{w}_{\mathrm{g}}(\mathrm{q})\right|^{2}+\frac{\sigma_{\mathrm{r}}^{2}}{\varepsilon_{\mathrm{x}_{2}}}},
$$

\subsection{Bit Error Rate Analysis}

In this subsection, the effect of phase noise on the bit error rate at the relay and at the destinations is characterized. 
At the relay, the upper bound and lower bound of the probability of error are given by Equation (18 and 19):

$$
\begin{aligned}
& \mathrm{P}_{\mathrm{e}, \text { Relay }}<\mathrm{P}_{\mathrm{e}, \text { Re lay }}^{\mathrm{U}}=\mathrm{Q}\left(\sqrt{2 \gamma_{1}[\mathrm{k}]}\right)+\mathrm{Q}\left(\sqrt{2 \gamma_{2}[\mathrm{k}]}\right) \\
& \mathrm{P}_{\mathrm{e}, \text { Relay }}>\mathrm{P}_{\mathrm{e}, \text { Relay }}^{\mathrm{L}}=\mathrm{Q}\left(\sqrt{2 \min \left(\gamma_{1}[\mathrm{k}], \gamma_{2}[\mathrm{k}]\right)}\right)
\end{aligned}
$$

The combined mixture $\gamma_{1}[\mathrm{k}], \gamma_{2}[\mathrm{k}]$ and $\min \left[\gamma_{1}[\mathrm{k}]\right.$, $\left.\gamma_{2}[\mathrm{k}]\right]$ are exponentially distributed random variables, with mean $\bar{\gamma}_{1}[\mathrm{k}], \bar{\gamma}_{2}[\mathrm{k}]$ and $\frac{\bar{\gamma}_{1}[\mathrm{k}] \bar{\gamma}_{2}[\mathrm{k}]}{\left(\bar{\gamma}_{1}[\mathrm{k}]+\bar{\gamma}_{2}[\mathrm{k}]\right)}$, respectively, where $\bar{\gamma}_{1}[\mathrm{k}]=\mathrm{E}\left[\gamma_{1}[\mathrm{k}]\right]$ and $\bar{\gamma}_{2}[\mathrm{k}]=\mathrm{E}\left[\gamma_{2}[\mathrm{k}]\right]$. Then, using (Simon and Alouini, 2005) Equation (5 and 6), the upper and lower bounds of $\mathrm{E}\left[\mathrm{P}_{\mathrm{e}, \text { Relay }}\right]$ are given by Equation (20):

$$
\begin{aligned}
& \varphi_{1}\left(\frac{\bar{\gamma}_{1}[\mathrm{k}] \bar{\gamma}_{2}[\mathrm{k}]}{\bar{\gamma}_{1}[\mathrm{k}]+\bar{\gamma}_{2}[\mathrm{k}]}\right)< \\
& \quad \mathrm{E}\left[\mathrm{P}_{\mathrm{e}, \text { Re lay }}\right]<\varphi_{1}\left(\bar{\gamma}_{1}[\mathrm{k}]\right)+\varphi_{1}\left(\bar{\gamma}_{2}[\mathrm{k}]\right)
\end{aligned}
$$

Where:

$$
\varphi_{1}(\bar{\gamma}[\mathrm{k}])=\frac{\left(1-\sqrt{\frac{\bar{\gamma}[\mathrm{k}]}{1+\bar{\gamma}[\mathrm{k}]}}\right)}{2}
$$

Using the upper and lower bounds from (18) and (19), the OFDM modulated instantaneous symbol error probability at the destination are derived as follows, The instantaneous symbol error probability $\mathrm{P}_{\mathrm{BC}, 1}$ of the $\mathrm{BC}$ at the destination, $\mathrm{S}_{1}$ from relay, $\mathrm{R}$ is $\mathrm{P}_{\mathrm{BC}, 1}=\mathrm{Q}\left(\sqrt{2 \gamma_{3}[\mathrm{k}]}\right)$ and the instantaneous symbol error probability $\mathrm{P}_{\mathrm{BC}, 2}$ of the $\mathrm{BC}$ at the destination, $\mathrm{S}_{2}$ from relay, $\mathrm{R}$ is $\mathrm{P}_{\mathrm{BC}, 2}=\mathrm{Q}\left(\sqrt{2 \gamma_{4}[\mathrm{k}]}\right)$.

Where Equation (21 and 22):

$$
\begin{aligned}
& \gamma_{3}[\mathrm{k}]=\frac{\left|\mathrm{w}_{\mathrm{h}}(\mathrm{k})\right|^{2}}{\frac{\pi \beta N T_{\mathrm{s}}}{3}\left|\sum_{\mathrm{q}=0, \mathrm{q} \neq \mathrm{k}}^{\mathrm{N}-1} \mathrm{w}_{\mathrm{h}}(\mathrm{q})\right|^{2}+\frac{\sigma_{\mathrm{n}}^{2}}{\varepsilon_{\mathrm{x}_{3}[\mathrm{k}]}}}, \\
& \gamma_{4}[\mathrm{k}]=\frac{\left|\mathrm{w}_{\mathrm{g}}(\mathrm{k})\right|^{2}}{\frac{\pi \beta N T_{\mathrm{s}}}{3}\left|\sum_{\mathrm{q}=0, \mathrm{q} \neq \mathrm{k}}^{\mathrm{N}-1} \mathrm{w}_{\mathrm{g}}(\mathrm{q})\right|^{2}+\frac{\sigma_{\mathrm{n}}^{2}}{\varepsilon_{\mathrm{x}_{3}[\mathrm{k}]}}}, \\
& \mathrm{i}=1,2
\end{aligned}
$$

The transmitted OFDM symbols from $S_{1}$ and $S_{2}$ undergoes two detections in cascade: one is the detection at relay, $\mathrm{R}$ whose error probability is $\mathrm{P}_{\mathrm{e}, \mathrm{Re}}$ ay and the other is the detection at $\mathrm{S}_{1}$ and $\mathrm{S}_{2}$, whose error probabilities are $\mathrm{P}_{\mathrm{BC}, 1}$ and $\mathrm{P}_{\mathrm{BC}, 2}$. Then the BER at destination $\mathrm{S}_{\mathrm{i}}$ is given by Equation (23):

$$
\begin{aligned}
\mathrm{P}_{\text {dest }, \mathrm{i}}= & \mathrm{P}_{\mathrm{e}, \text { Re lay }}\left(1-\mathrm{P}_{\mathrm{BC}, \mathrm{i}}\right)+\left(1-\mathrm{P}_{\mathrm{e}, \text { Re lay }}\right) \mathrm{P}_{\mathrm{BC}, \mathrm{i}} \\
& \mathrm{i}=1,2
\end{aligned}
$$

Averaging $\mathrm{P}_{\text {dest, } 1}$ and $\mathrm{P}_{\text {dest,2 }}$ gives the instantaneous $\mathrm{BER}$ at the destination is given by Equation (24):

$$
\mathrm{P}_{\text {dest }}=\frac{1}{2} \sum_{\mathrm{i}=1}^{2} \mathrm{P}_{\text {dest, } \mathrm{i}}
$$

The upper and lower bounds of $\mathrm{P}_{\text {dest }}$ are derived in the following theorem.

\section{Theorem 1}

The overall upper bound $\mathrm{E}\left[\mathrm{P}_{\text {dest }}^{\mathrm{U}}\right]$ and lower bound $\mathrm{E}\left[\mathrm{P}_{\text {dest }}^{\mathrm{L}}\right]$ at the destination are given respectively by Equation (25 and 26):

$$
\begin{aligned}
& \mathrm{E}\left[\mathrm{P}_{\text {dest }}\right]<\mathrm{E}\left[\mathrm{P}_{\text {dest }}^{\mathrm{U}}\right] \stackrel{\text { def }}{=} \\
& \quad \varphi_{1}\left(\bar{\gamma}_{1}[\mathrm{k}]\right)+\varphi_{1}\left(\bar{\gamma}_{2}[\mathrm{k}]\right)+\frac{1}{2} \Xi\left(\bar{\gamma}_{1}[\mathrm{k}], \bar{\gamma}_{2}[\mathrm{k}], \bar{\gamma}_{3}[\mathrm{k}], \bar{\gamma}_{4}[\mathrm{k}]\right) \\
& \mathrm{E}\left[\mathrm{P}_{\text {dest }}\right]>\mathrm{E}\left[\mathrm{P}_{\text {dest }}^{\mathrm{L}}\right] \stackrel{\text { def }}{=} \\
& \quad \varphi_{1}\left(\frac{\bar{\gamma}_{1}[\mathrm{k}] \bar{\gamma}_{2}[\mathrm{k}]}{\bar{\gamma}_{1}[\mathrm{k}]+\bar{\gamma}_{2}[\mathrm{k}]}\right)+\frac{1}{2} \Xi\left(\bar{\gamma}_{1}[\mathrm{k}], \bar{\gamma}_{2}[\mathrm{k}], \bar{\gamma}_{3}[\mathrm{k}], \bar{\gamma}_{4}[\mathrm{k}]\right)
\end{aligned}
$$

Where Equation (27):

$$
\begin{aligned}
\Xi & \left(\bar{\gamma}_{1}[\mathrm{k}], \bar{\gamma}_{2}[\mathrm{k}], \bar{\gamma}_{3}[\mathrm{k}], \bar{\gamma}_{4}[\mathrm{k}]\right)= \\
& \boldsymbol{\varphi}_{1}\left(\bar{\gamma}_{3}[\mathrm{k}]\right)+\boldsymbol{\varphi}_{1}\left(\bar{\gamma}_{4}[\mathrm{k}]\right)-2 \boldsymbol{\varphi}_{1}\left(\bar{\gamma}_{1}[\mathrm{k}]\right) \boldsymbol{\varphi}_{1}\left(\bar{\gamma}_{4}[\mathrm{k}]\right) \\
& -2 \boldsymbol{\varphi}_{1}\left(\bar{\gamma}_{2}[\mathrm{k}]\right) \boldsymbol{\varphi}_{1}\left(\bar{\gamma}_{3}[\mathrm{k}]\right)-\boldsymbol{\varphi}_{2}\left(\bar{\gamma}_{1}[\mathrm{k}], \bar{\gamma}_{3}[\mathrm{k}]\right)-\boldsymbol{\varphi}_{2}\left(\bar{\gamma}_{2}[\mathrm{k}], \bar{\gamma}_{4}[\mathrm{k}]\right) \\
& -\sqrt{\frac{\bar{\gamma}_{2}[\mathrm{k}]}{\bar{\gamma}_{2}[\mathrm{k}]+1}} \boldsymbol{\varphi}_{2}\left(\frac{\bar{\gamma}_{1}[\mathrm{k}]\left(1+\bar{\gamma}_{2}[\mathrm{k}]\right)}{\bar{\gamma}_{2}[\mathrm{k}]}, \gamma_{3}[\mathrm{k}]\right) \\
& -\sqrt{\frac{\bar{\gamma}_{1}[\mathrm{k}]}{\bar{\gamma}_{1}[\mathrm{k}]+1}} \boldsymbol{\varphi}_{2}\left(\frac{\bar{\gamma}_{2}[\mathrm{k}]\left(1+\bar{\gamma}_{1}[\mathrm{k}]\right)}{\bar{\gamma}_{1}[\mathrm{k}]}, \gamma_{4}[\mathrm{k}]\right) \\
\varphi_{2}\left(\mathrm{~b}_{1}[\mathrm{k}], \mathrm{b}_{2}[\mathrm{k}]\right)= & \frac{1}{2}\left[\frac{\varphi_{1}\left(\mathrm{~b}_{1}[\mathrm{k}], \mathrm{b}_{2}[\mathrm{k}]\right)}{\pi}-\frac{\delta_{1}[\mathrm{k}]}{\pi}\left(\frac{\pi}{2}+\tan ^{-1} \varsigma_{1}[\mathrm{k}]\right)\right] \\
+ & \frac{1}{2}\left[\frac{\varphi_{2}\left(\mathrm{~b}_{1}[\mathrm{k}], \mathrm{b}_{2}[\mathrm{k}]\right)}{\pi}-\frac{\delta_{2}[\mathrm{k}]}{\pi}\left(\frac{\pi}{2}+\tan ^{-1} \varsigma_{2}[\mathrm{k}]\right)\right]
\end{aligned}
$$


where,

$$
\delta_{\mathrm{i}}[\mathrm{k}]=\sqrt{\frac{\mathrm{b}_{\mathrm{i}}[\mathrm{k}]}{2+\mathrm{b}_{1}[\mathrm{k}]}} \operatorname{sgn}\left(\varphi_{\mathrm{i}}\left(\mathrm{b}_{1}[\mathrm{k}], \mathrm{b}_{2}[\mathrm{k}]\right)\right),
$$

$\varsigma_{\mathrm{i}}[\mathrm{k}]=-\delta_{\mathrm{i}}[\mathrm{k}] \cot \left(\varphi_{\mathrm{i}}\left(\mathrm{b}_{1}[\mathrm{k}], \mathrm{b}_{2}[\mathrm{k}]\right)\right) \quad$ for $\quad \mathrm{i} \quad=1,2 \quad$ with

$\varphi_{1}\left(\mathrm{~b}_{1}[\mathrm{k}], \mathrm{b}_{2}[\mathrm{k}]\right)=\frac{\pi}{2}-\tan ^{-1}\left(\frac{\mathrm{b}_{2}[\mathrm{k}]}{\mathrm{b}_{1}[\mathrm{k}]}\right)$

and

$\varphi_{2}\left(\mathrm{~b}_{1}[\mathrm{k}], \mathrm{b}_{2}[\mathrm{k}]\right)=\tan ^{-1}\left(\frac{\mathrm{b}_{2}[\mathrm{k}]}{\mathrm{b}_{1}[\mathrm{k}]}\right)$ and $\operatorname{sgn}($.$) is the sign$ function.

\subsection{Outage Probability Analysis}

The outage probability for the $\mathrm{k}^{\text {th }}$ subcarrier for the given data rate $R_{d}$ is given by Equation (28):

$$
\mathrm{P}\left(\mathrm{R}_{\mathrm{d}}\right)=\mathrm{P}\left(\log \left(1+\gamma_{3}[\mathrm{k}]\right)<\mathrm{R}_{\mathrm{d}}\right)=\mathrm{P}\left(\gamma_{3}[\mathrm{k}]<2^{\mathrm{R}_{\mathrm{d}}}-1\right)
$$

For simplicity, the SINR $\gamma_{3}[\mathrm{k}]$ expression in (21) can be modeled as $\mathrm{X} /(\mathrm{aY}+\mathrm{b})$.

Where:

$$
\mathrm{X}=\left|\mathrm{w}_{\mathrm{h}}(\mathrm{k})\right|^{2}, \mathrm{Y}=\left|\sum_{\mathrm{q}=0, \mathrm{q} \neq \mathrm{k}}^{\mathrm{N}-1} \mathrm{w}_{\mathrm{h}}(\mathrm{q})\right|^{2}, \mathrm{~b}=\frac{\pi \beta \mathrm{T}_{\mathrm{s}}}{3} \text { and } \mathrm{c}=\frac{\sigma_{\mathrm{n}}^{2}}{\varepsilon_{\mathrm{x}_{3}}}
$$

Therefore Equation (29):

$$
\mathrm{P}\left(\mathrm{R}_{\mathrm{d}}\right) \leq \mathrm{P}\left(\frac{\mathrm{X}}{\mathrm{bY}+\mathrm{c}}<2^{\mathrm{R}_{\mathrm{d}}}-1\right)
$$

In defining the substitution, $X$ is an exponential random variable with parameter $\lambda_{\mathrm{x}}$ and $\mathrm{Y}$ is Gamma distributed random variable with scale parameter, $\frac{\mathrm{N}}{\mathrm{v}}$ and shape parameter, v. The Cumulative distribution Function for the random variable $U=\frac{X}{b Y+C}$ with exponentially distributed $\mathrm{X}$ and gamma distributed $\mathrm{Y}$ is given by Equation (30):

$$
\begin{aligned}
F_{U}(u, b, c) & =P\left(\frac{X}{b Y+c}<u\right) \\
& =\int_{0}^{\infty} \int_{0}^{b y u+c u)} f_{X}(x) f_{Y}(y) d x d y
\end{aligned}
$$

Solving the integration using the appropriate PDF functions we can get:

$$
F_{U}(u, b, c)=1-\exp \left(-\lambda_{x} c u\right)\left(1+\frac{N \lambda_{x} b u}{v}\right)^{-v}
$$

Using (31), the outage probability is upper bounded by Equation (31):

$$
\mathrm{p}\left(\mathrm{R}_{\mathrm{d}}\right) \leq 1-\mathrm{e}^{-\left(2^{\mathrm{R}_{\mathrm{d}}-1}\right) \sigma_{\mathrm{x}}^{2} / \varepsilon_{\mathrm{x}}}\left(1+\frac{\left(2^{\mathrm{R}_{\mathrm{d}}}-1\right) \pi \beta \mathrm{NT}}{3 \mathrm{v}}\right)^{-\mathrm{v}}
$$

At high SNR, it can be approximated as Equation (32):

$$
\mathrm{p}\left(\mathrm{R}_{\mathrm{d}}\right)=\frac{\left(2^{\mathrm{R}_{\mathrm{d}}}-1\right) \pi \beta N \mathrm{~T}_{\mathrm{s}}}{3 \mathrm{v}}
$$

Where, $\mathrm{N} \mathrm{T}_{\mathrm{s}}$ is the PHN Level Percentage (PLP).

\section{RESULTS}

Monte Carlo simulation is carried out in MATLAB to evaluate the performance of OFDM based bidirectional relay network in the presence of phase noise. The simulation parameters are tabulated in Table 1. Figure 2 and 3 shows the bit error rate performance with various phase noise bandwidths. Figure 4 shows the upper and lower bounds of the average symbol error probability during the MAC phase. Figure 5 illustrates the overall End-to-End average symbol error probability with the upper and lower bounds. In Fig. 6, the Outage performance for both analytical and simulation results is also analyzed during the End-to-End transmission.

\section{DISCUSSION}

Figure 2 shows the average BER performance of OFDM based bidirectional relay networks for various phase noise bandwidths assuming number of subcarriers $\mathrm{N}$ $=1024$. It is observed that the probability of error increases with the increase in phase noise bandwidth up to the maximum subcarrier spacing of $20 \mathrm{KHz}$. At low SNR, there is no much deviation in the probability of error with reasonable increase in the PHN bandwidth. In contrast, during the high SNR regime, the deviation in the probability of error is much high i.e., for the probability error of $10^{-2}, 25 \mathrm{~dB}$ more SNR is needed when the PHN bandwidth is $10 \mathrm{KHz}$. Further it can be noticed that when the phase noise bandwidth is greater than the subcarrier spacing, the system performance deteriorates. 
V.N. Senthil Kumaran et al. / American Journal of Applied Sciences 10 (11): 1335-1344, 2013

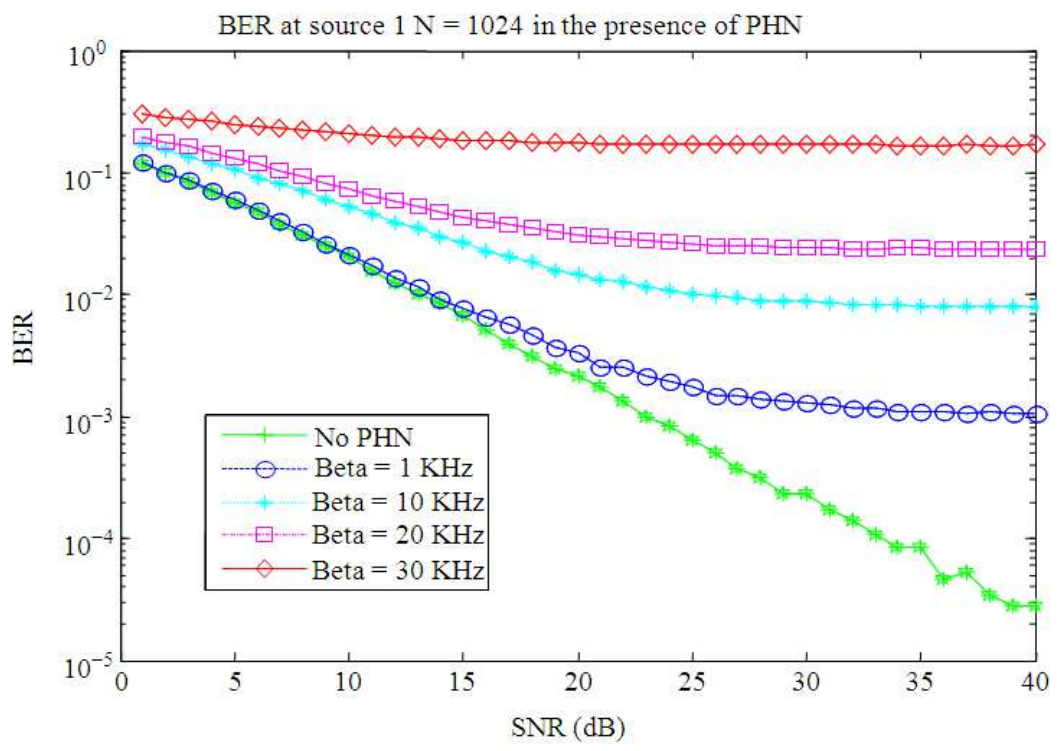

Fig. 2. End-to-End average BERs of the OFDM based PLNC protocol with phase noise at $N=1024$

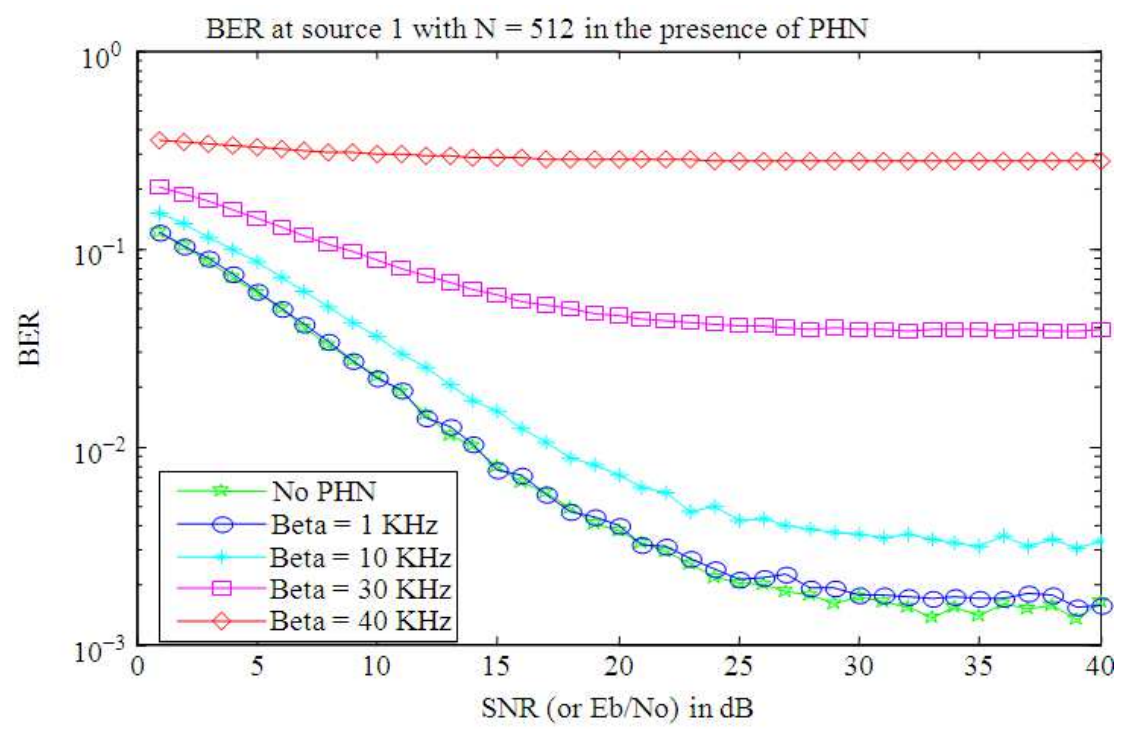

Fig. 3. End-to-End average BERs of the OFDM based PLNC with phase noise at $\mathrm{N}=512$

Table 1. Simulation parameters

\begin{tabular}{ll}
\hline OFDM signal bandwidth & $20 \mathrm{MHz}$ \\
Sampling interval & 50 nano seconds \\
FFT size & 512,1024 \\
Sub carrier spacing & $40 \mathrm{KHz}, 20 \mathrm{KHz}$ \\
Cyclic Prefix length & 16 \\
Phase noise bandwidths & $1 \mathrm{KHz}, 10 \mathrm{KHz}, 20 \mathrm{KHz}$, \\
& $30 \mathrm{KHz}, 40 \mathrm{KHz}$ \\
\hline
\end{tabular}

Figure 3 shows the BER performance of OFDM based bidirectional relay networks with the subcarrier spacing of $40 \mathrm{KHz}$, OFDM signal bandwidth, $\mathrm{BW}=$ $20 \mathrm{MHz}$ and number of sub carriers $\mathrm{N}=512$. Finally we conclude that by reducing the phase noise bandwidth or by increasing the number of sub carriers we can achieve the best performance. 


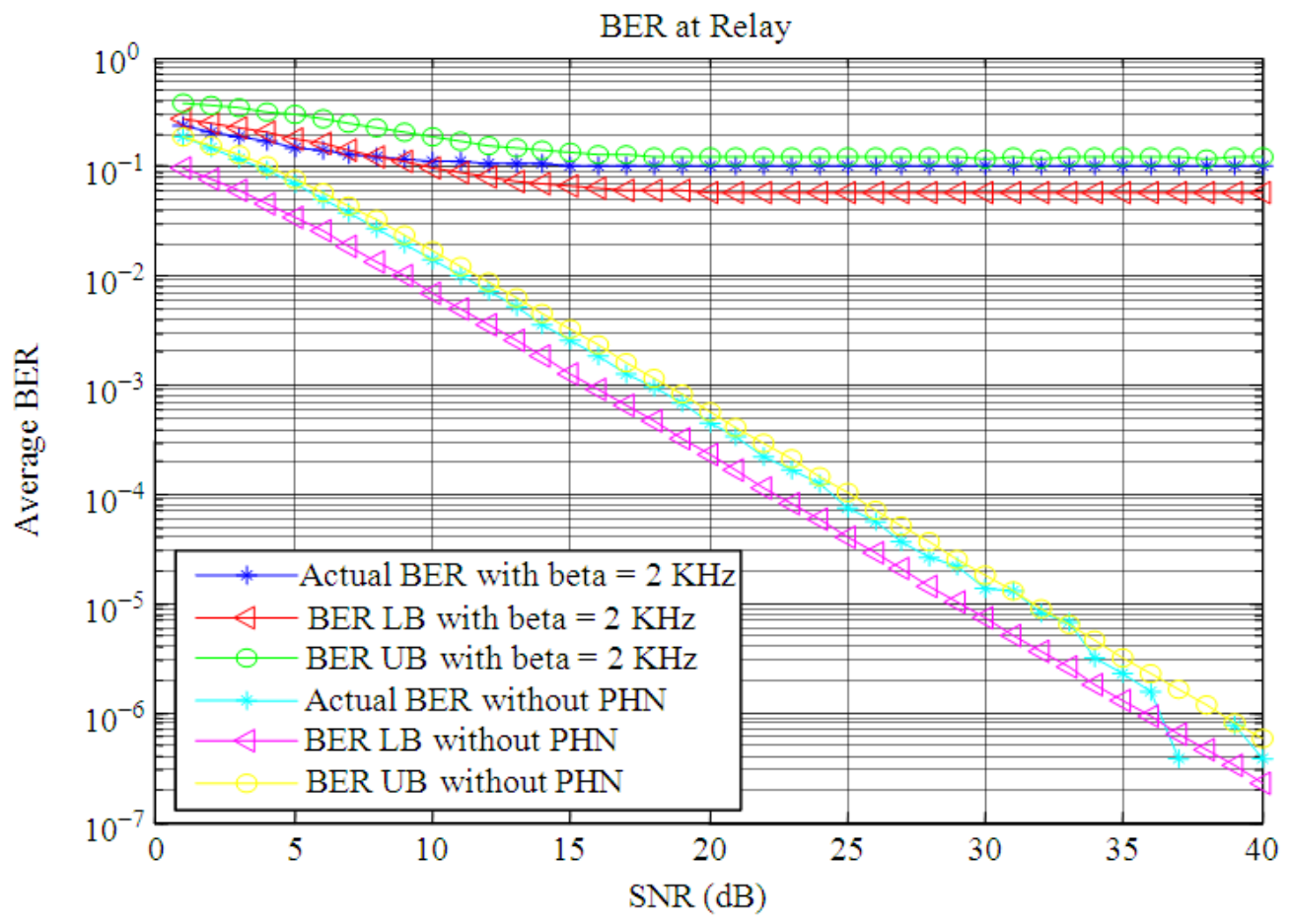

Fig. 4. Average symbol error probability with upper and lower bounds in the presence of phase noise at the Relay with $\mathrm{N}=1024$

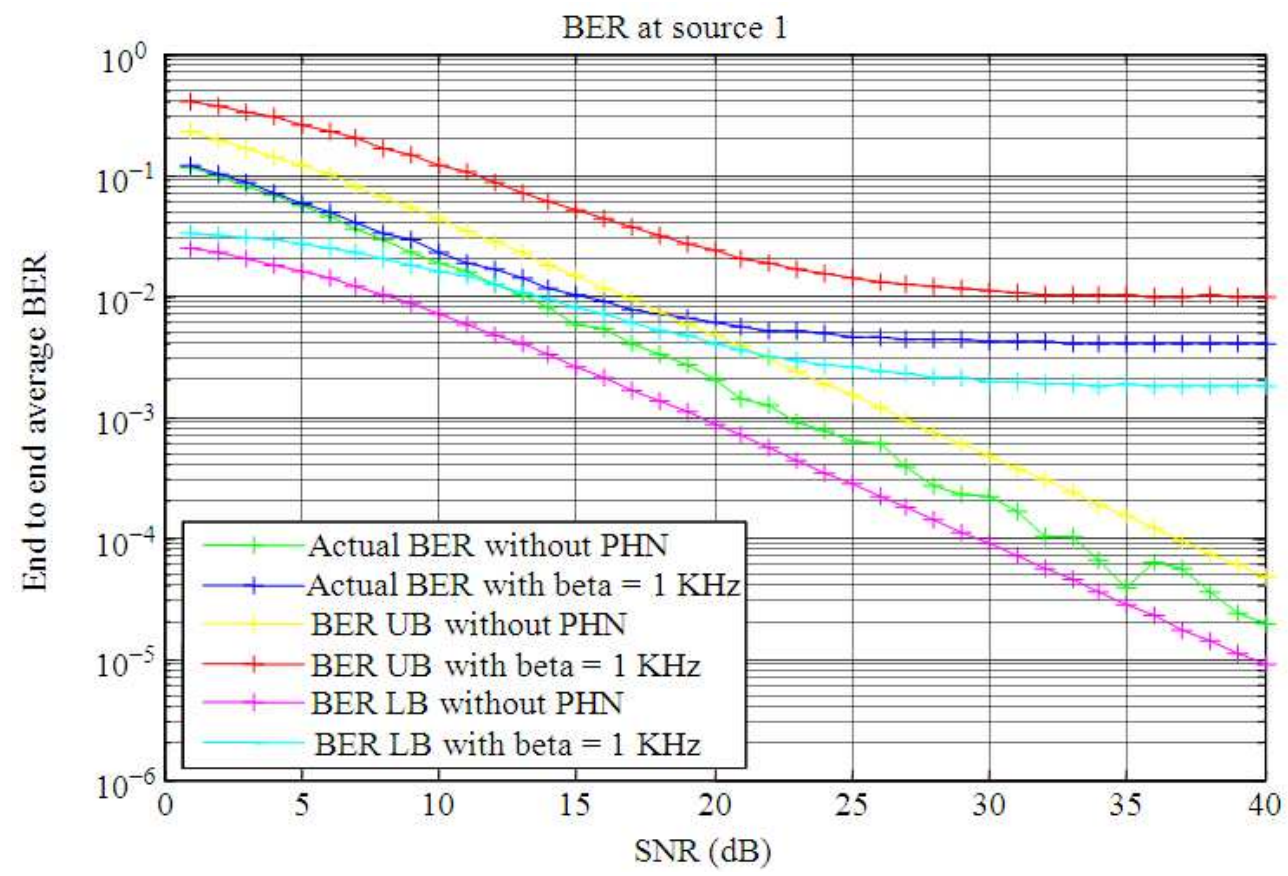

Fig. 5. End-to-End Average symbol error probability with upper and lower bounds in the presence of phase noise at the destination node $\mathrm{S}_{1}$ with $\mathrm{N}=1024$ 


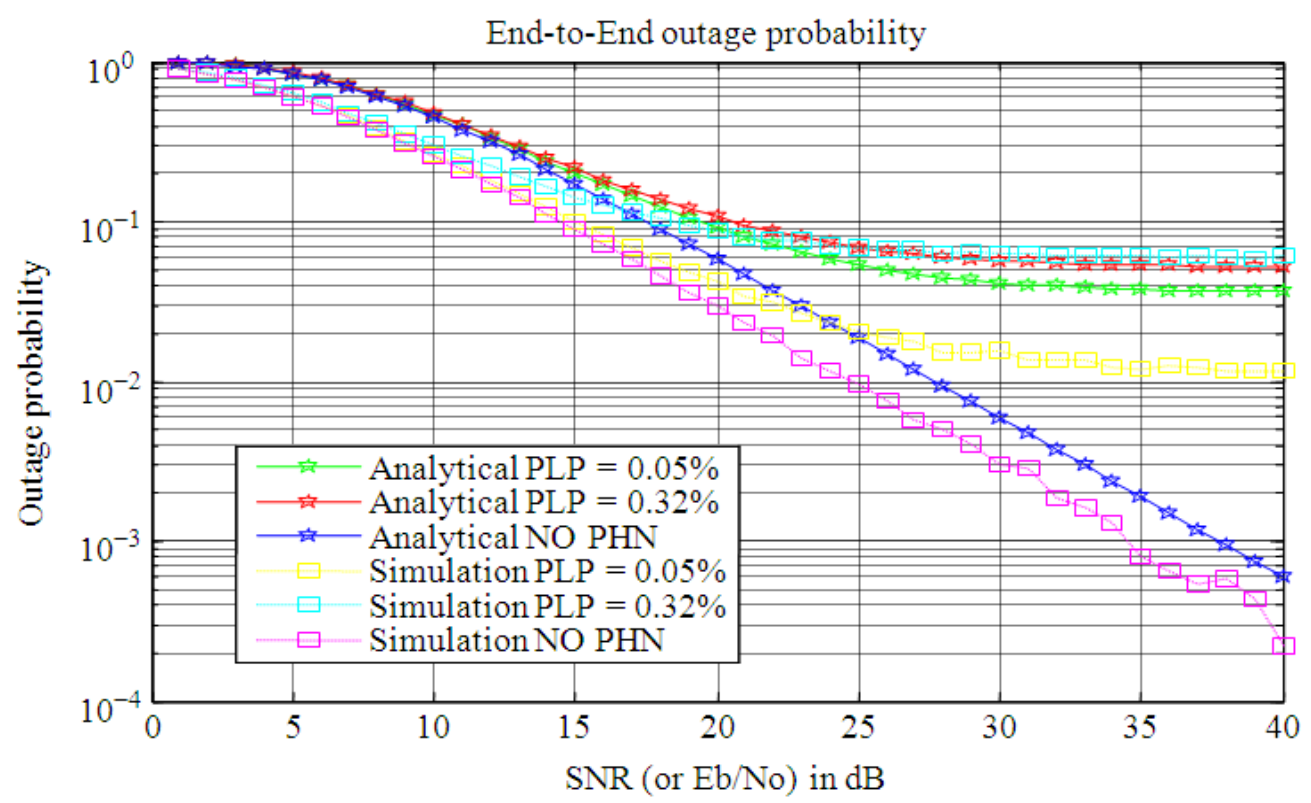

Fig. 6. End-to-End transmission Outage probability for different PHN levels. Solid lines are the upper bound results while dashed lines are the simulations $\mathrm{N}=512, \mathrm{R}_{\mathrm{d}}=2$

Figure 4 shows the average symbol error probability $\mathrm{E}\left[\mathrm{P}_{\mathrm{e}, \mathrm{Re} \text { lay }}\right]$ at Relay, $\mathrm{R}$ for phase noise bandwidth of $2 \mathrm{KHz}$ with upper bound, $\mathrm{E}\left[\mathrm{P}_{\mathrm{e}, \mathrm{U} \text { lay }}^{\mathrm{U}}\right]$ and lower bound, $\mathrm{E}\left[\mathrm{P}_{\mathrm{e}, \mathrm{Re} \text { lay }}^{\mathrm{L}}\right]$. Figure 5 shows the average symbol error probability $\mathrm{E}\left[\mathrm{P}_{\mathrm{EtoE}}\right]$ of the $\mathrm{BC}$ at the destinations $\mathrm{S}_{1}$ and $\mathrm{S}_{2}$ with upper bound, $\mathrm{E}\left[\mathrm{P}_{\mathrm{EtoE}}^{\mathrm{U}}\right]$ and lower bound, $\mathrm{E}\left[\mathrm{P}_{\mathrm{EtoE}}^{\mathrm{L}}\right]$.

The outage probability of the End-to-End transmission case for different PLPs is presented in Fig. 6. As it can seen from the figure, at high SNR the analytical result is upper bounded.

\section{CONCLUSION}

The impact of phase noise on OFDM based bidirectional relay network has been presented. Simulation results show that the phase noise causes severe distortion in the OFDM based bidirectional relay system. The BER degradation is calculated for various PHN levels. We have obtained both the upper and lower bounds for the MAC and end-to-end BER. The analytical and confirming simulation results of the End-to-end outage probability is obtained. We are further investigating the various PHN compensation algorithms to improve the performance of the OFDM based relay networks in the presence of phase noise.

\section{ACKNOWLEDGEMENT}

The researchers are very much thankful to the Management of Thiagarajar College of Engineering for their support and assistance to carry out this research.

\section{REFERENCES}

Ahlswede, R., N. Cai, S.R. Li and R.W. Yeung, 2000. Network information flow. IEEE Trans. Inform. Theory, 46: 1204-1217. DOI: 10.1109/18.850663

Armada, A.G., 2001. Understanding the effects of phase noise in Orthogonal Frequency Division Multiplexing (OFDM). IEEE Trans. Broadcast., 47: 153-159. DOI: 10.1109/11.948268

Ding, Z., K.K. Leung, D.L. Goeckel and D. Towsley, 2009. On the study of network coding with diversity. IEEE Trans. Wireless Commun., 8: 12471259. DOI: 10.1109/TWC.2009.07051022

Gao, F., R. Zhang and Y.C. Liang, 2009. Channel estimation for OFDM modulated two-way relay networks. IEEE Trans. Signal Process., 57: 44434455. DOI: 10.1109/TSP.2009.2026537

MinChul, J. and I.M. Kim, 2010. Error performance analysis of BPSK modulation in physical-layer network-coded bidirectional relay networks. IEEE Trans. Commun., 58: 2770-2775. DOI: 10.1109/TCOMM.2010.082010.090256 
Petrovic, D., W. Rave and G. Fettweis, 2007. Effects of phase noise on OFDM systems with and without PLL: Characterization and compensation. IEEE Trans. Commun., 55: 1607-1616. DOI: 10.1109/TCOMM.2007.902593

Rabiei, P., W. Namgoong and N. Al-Dhahir, 2008. Frequency domain joint channel and phase noise estimation in OFDM WLAN systems. Proceedings of the 42nd Asilomar Conference on Signals, Systems and Computers, Oct. 26-29, IEEE Xplore Press, Pacific Grove, CA., pp: 928-932. DOI: 10.1109/ACSSC.2008.5074547

Rabiei, P., W. Namgoong and N. Al-Dhahir, 2011. On the performance of OFDM-based amplify-andforward relay networks in the presence of phase noise. IEEE Trans. Commun., 59: 1458-1566. DOI: 10.1109/TCOMM.2011.030411.100206

Simon, M.K. and M.S. Alouini, 2005. Digital Communication over Fading Channels. 2nd Edn., John Wiley and Sons, New York, ISBN-10: 0471715239, pp: 936.

Tomba, L., 1998. On the effect of Wiener phase noise in OFDM systems. IEEE Trans. Commun., 46: 580583. DOI: $10.1109 / 26.668721$
Vaze, R., K.T. Truong, S. Weber and R.W. Heath, 2011. Two-way Transmission capacity of wireless Ad-hoc networks. IEEE Trans. Wireless Commun., 10: 19661975. DOI: 10.1109/TWC.2011.041311.101488

Wang, G., F. Gao, X. Zhang and C. Tellambura, 2010. Superimposed training-based joint CFO and channel estimation for CP-OFDM modulated two-way relay networks. EURASIP J. Wireless Commun. Network. DOI: $10.1155 / 2010 / 403936$

$\mathrm{Wu}$, S. and Y. Bar-Ness, 2004. OFDM systems in the presence of phase noise: Consequences and solutions. IEEE Trans. Commun., 52: 1988-1996. DOI: 10.1109/TCOMM.2004.836441

Zhang, S., F. Gao, C. Pei and X. He, 2013. Segment training based individual channel estimation in oneway relay network with power allocation. IEEE Trans. Wireless Commun., 12: 1300-1309. DOI: 10.1109/TWC.2013.013013.120768

Zhang, S., S.C. Liew and P.P. Lam, 2006. Hot topic: Physical-layer network codin. Proceedings of the 12th Annual International Conference on Mobile Computing and Networking, Sept. 24-29, ACM Press, Los Angeles, CA, USA., pp: 358-365. DOI: 10.1145/1161089.1161129 